\title{
Factors influencing healthcare provider respondent fatigue answering a globally administered in-app survey
}

\author{
Vikas N O'Reilly-Shah ${ }^{\text {Corresp. } 1,2}$ \\ 1 Department of Anesthesiology, Emory University, Atlanta, Georgia, United States \\ 2 Department of Anesthesiology, Children's Healthcare of Atlanta, Atlanta, Georgia, United States \\ Corresponding Author: Vikas N O'Reilly-Shah \\ Email address: voreill@emory.edu
}

Background: Respondent fatigue, also known as survey fatigue, is a common problem in the collection of survey data. Factors that are known to influence respondent fatigue include survey length, survey topic, question complexity, and open-ended question type. There is a great deal of interest in understanding the drivers of physician survey responsiveness due to the value of information received from these practitioners. With the recent explosion of mobile smartphone technology, it has been possible to obtain survey data from users of mobile applications (apps) on a question-by-question basis. The author obtained basic demographic survey data as well as survey data related to an anesthesiology-specific drug called sugammadex and leveraged nonresponse rates to examine factors that influenced respondent fatigue.

Methods: Primary data were collected between December 2015 and February 2017. Surveys and in-app analytics were collected from global users of a mobile anesthesia calculator app. Key independent variables were user country, healthcare provider role, rating of importance of the app to personal practice, length of time in practice, and frequency of app use. Key dependent variable was the metric of respondent fatigue.

Results: Provider role and World Bank country income level were predictive of the rate of respondent fatigue for this in-app survey. Importance of the app to the provider and length of time in practice were moderately associated with fatigue. Frequency of app use was not associated. This study focused on a survey with a topic closely related to the subject area of the app. Respondent fatigue rates will likely change dramatically if the topic does not align closely.

Discussion: Although apps may serve as powerful platforms for data collection, responses rates to in-app surveys may differ on the basis of important respondent characteristics. Studies should be carefully designed to mitigate fatigue as well as powered with the understanding of the respondent characteristics that may have higher rates of respondent fatigue. 


\section{Title}

2

3 Factors influencing healthcare provider respondent fatigue answering a globally administered in-

4 app survey

\section{Corresponding Author}

6

7 Vikas O'Reilly-Shah, MD, PhD

8 Assistant Professor of Anesthesiology

9 Emory University and Children's Healthcare of Atlanta

10 3B South

111364 Clifton Road NE

12 Atlanta, GA 30322

13 voreill@emory.edu

$14+1-615-335-3808$

15

16

17

18 


\section{Abstract}

Background: Respondent fatigue, also known as survey fatigue, is a common problem in the collection of survey data. Factors that are known to influence respondent fatigue include survey length, survey topic, question complexity, and open-ended question type. There is a great deal of interest in understanding the drivers of physician survey responsiveness due to the value of information received from these practitioners. With the recent explosion of mobile smartphone technology, it has been possible to obtain survey data from users of mobile applications (apps) on a question-by-question basis. The author obtained basic demographic survey data as well as survey data related to an anesthesiology-specific drug called sugammadex and leveraged nonresponse rates to examine factors that influenced respondent fatigue.

Methods: Primary data were collected between December 2015 and February 2017. Surveys and

31 in-app analytics were collected from global users of a mobile anesthesia calculator app. Key

32 independent variables were user country, healthcare provider role, rating of importance of the

33 app to personal practice, length of time in practice, and frequency of app use. Key dependent

34 variable was the metric of respondent fatigue.

35 Results: Provider role and World Bank country income level were predictive of the rate of 36 respondent fatigue for this in-app survey. Importance of the app to the provider and length of

37 time in practice were moderately associated with fatigue. Frequency of app use was not associated. This study focused on a survey with a topic closely related to the subject area of the

39 app. Respondent fatigue rates will likely change dramatically if the topic does not align closely.

40 Discussion: Although apps may serve as powerful platforms for data collection, responses rates

41 to in-app surveys may differ on the basis of important respondent characteristics. Studies should 
42 be carefully designed to mitigate fatigue as well as powered with the understanding of the

43 respondent characteristics that may have higher rates of respondent fatigue.

44

45

46 


\section{Introduction}

The explosion of smartphone technology (Rivera \& van der Meulen) that has

accompanied the digital revolution brings opportunities for research into human behaviour at an unprecedented scale. Mobile analytics, ("Introducing Amazon Mobile Analytics", "Google Analytics", "Analytics for Xamarin", "Microsoft Azure”) along with tools that can supplement these analytics with survey data, (Xiong et al.; O'Reilly-Shah \& Mackey, 2016) have become easy to integrate into the millions of available apps in public app stores.("Number of apps available in leading app stores 2015 | Statistic") Overall growth in app availability and use has been accompanied by concomitant growth in the mobile health (mHealth) space. (Akter \& Ray, 2010; Liu et al., 2011; Ozdalga, Ozdalga \& Ahuja, 2012) mHealth is an established MeSH entry term that broadly describes efforts in the area of mobile-based health information delivery, although a reasonable argument can be made that it includes the collection of analytics and metadata from consumers of this information as well. ("Definitions of mHealth," 2012, "MeSH Browser") Surveys are a critical supplement to these analytics and metadata because they provide direct information about user demographic characteristics as well as the opinion information that researchers are most interested in understanding. Much of the mHealth literature has made use of surveys deployed them via Web-based online surveys (e.g. via REDCap (Harris et al., 2009) or via SurveyMonkey (SurveyMonkey, Inc, San Mateo, CA, USA)) rather than inapp surveys. However, mobile applications that are used by specialist populations create an opportunity for collection of information from a targeted group via in-app surveys, and tools are being developed to assess the quality of these health mobile apps for even better targeting.

68 (Stoyanov et al., 2015) 

of survey data. (Whelan; Ben-Nun, 2008) It refers to the situation in which respondents give less

71 thoughtful answers to questions in the later parts of a survey, or prematurely terminate

72 participation. (Whelan; Ben-Nun, 2008; Hochheimer et al., 2016) This may be detected when

73 there is straight-line answering, where the respondent chooses e.g. the first option of a multiple

74 choice survey for multiple question in a row. It may also be present if the respondent leaves text

75 response fields blank or if the respondent chooses the "default" response on a slider bar. Finally,

76 fatigue may be present if the respondent fails to complete the survey. (Ben-Nun, 2008;

77 Hochheimer et al., 2016) Factors that are known to influence respondent fatigue include survey

78 length, survey topic, question complexity, and question type (open-ended questions tend to

79 induce more fatigue). (Ben-Nun, 2008) Respondent fatigue lowers the quality of data collected

80 for later questions in the survey and can introduce bias into studies, including nonresponse bias.

81 (“JSM 2016 Online Program”, “2015 Joint Statistical Meetings - Statistics: Making Better

82 Decisions. - Seattle, Washington”)

83 There is a great deal of interest in understanding the drivers of physician survey

84 responsiveness due to the value of information received from these practitioners. (Kellerman,

85 2001; Cull et al., 2005; Nicholls et al., 2011; Glidewell et al., 2012; Cook et al., 2016) These

86 studies typically looked at overall response rate rather than respondent fatigue. The collection of

87 survey data in mobile apps may be collected on a question-by-question basis. (O’Reilly-Shah \&

88 Mackey, 2016) While this increases the amount of data available to researchers, it also increases

89 the risk of obtaining incomplete survey data as it may become more commonplace for users to

90 discontinue study participation in the middle of a survey. 
While incomplete survey data reduces the quality of a dataset, it also provides an

92 opportunity to study respondent fatigue directly. In the course of a study of more than 10,000

93 global users of a mobile anesthesia calculator app, (O’Reilly-Shah, Easton \& Gillespie, 2017) the

94 author obtained basic demographic survey data as well as survey data related to an

95 anesthesiology-specific drug called sugammadex. Nonresponse rates were leveraged to examine

96 factors that influenced respondent fatigue.

\section{Methods} deployed a mobile anesthesia calculator app fitted with the Survalytics platform. (O'Reilly-Shah \& Mackey, 2016) A screenshot of the app interface is provided in Figure S1. The calculator is

101 designed to provide age and weight based clinical decision support for anesthetic management,

102 including information about airway equipment, emergency management, drug dosing, and nerve-

103 blocks. Survalytics enables cloud-based delivery of survey questions and storage of both survey

104 responses and app "analytics" using an Amazon (Seattle, WA) Web Services database. Here,

105 analytics is used to mean collected and derived metadata including app use frequency, in-app

106 activity, device location and language, and time of use. Two surveys were deployed: one to

107 collect basic user demographic information, and another to characterize attitudes and adverse

108 event rates related to the drug sugammadex. These surveys are available for review in the

109 Supplementary Data in Tables S1 and S2. Survey questions appear immediately after launch of

110 the app, with a "Not Now/Answer Later" button, so if the user is needing to reference the app for

111 emergency purposes then they can immediately go to the calculator without being forced to the

112 answer the survey. Although data collection is ongoing, the study period for this work is limited

113 to data collected between December 2015 and February 2017. The sugammadex survey was 
114 deployed in March 2016. The results of the sugammadex survey itself are beyond the scope of

115 the present analysis, although preliminary results have been presented at a meeting and in

116 correspondence. (O’Reilly-Shah, 2016; O’Reilly-Shah et al., 2017)

117 Raw data from the DynamoDB database were downloaded and processed using CRAN R

118 (R Core Team, Vienna, Austria) v3.3 in the RStudio (RStudio Team, Boston, MA) environment.

119 (South, 2011; Arel-Bundock, 2014; Ooms, 2014; R Core Team, 2015; RStudio-Team, 2015)

120 User country was categorized using public World Bank classification of country income level.

121 ("World Bank Country Incomes") In cases where users were active in more than one country, the

122 country in which the most app uses were logged was taken as the primary country. Detailed

123 information about the Survalytics package, the data collected for this study, and the approach to

124 calculation of frequency of app use can be found in the Supplementary Appendix.

125 The study was reviewed and approved by the Emory University Institutional Review

126 Board \#IRB00082571. This review included a finding by the FDA that Anesthesiologist falls

127 into the category of "enforcement discretion" as a medical device, meaning that, at present, the

128 FDA does not intend to enforce requirements under the FD\&C Act. (FDA \& U.S. Department of

129 Health and Human Services, Food and Drug Administration, Center for Devices and

130 Radiological Health, Center for Biologics Evaluation and Research, 2015)

131 Statistical Methods

132 Subjects were categorized as "fatigued" or "not fatigued" according to whether they

133 responded to the first unbranched sugammadex survey question (Table S2, Q-03) but did not

134 complete the survey to the last question (Table S2, Q-10). This classification was used to

135 perform logistic regression analysis against several independent variables, including provider

136 role, frequency of app use, country income level, rating of app importance, and length of time in 
137 practice. Some of these were objectively gathered as metadata collected via the Survalytics

138 package. Others were gathered from users as part of the basic demographic (Table S1).

139 Results

140 There was a consistent rate of data collection throughout the study period (Figure 1).

141 Following successful study launch in December 2015, the sugammadex survey was put into the

142 field in March 2016. Responses to this survey were consistently collected throughout the study

143 period, at a rate of approximately 179 total responses per day (green line, magnified 10x). There

144 was a demonstrable and consistent rate of respondent fatigue, leading to the observed decrease in

145 the rate of responses to the first unbranched question of the sugammadex survey (Q-03, blue line,

146 magnified 50x, 16 responses collected per day) versus the last (Q-10, purple line, magnified 50x,

14711 responses collected per day).

148 The overall rate of respondent fatigue was $34.3 \%(\mathrm{~N}=5991)$. Respondent fatigue then

149 analyzed by several respondent characteristics. Some of these characteristics were based on self-

150 reported data collected in the baseline survey (provider type, importance of app to personal

151 practice), while others were based on objective data (user location, frequency of app use).

152 Results of univariable logistic regression analysis are shown in Table 1.

153 Provider role was an excellent predictor of the rate of respondent fatigue (Figure 2, N =

$1545333, \mathrm{p}<0.001)$. Physicians and physician trainees were most likely to complete the

155 sugammadex survey, while technicians and respiratory therapists were least likely to do so. Main

156 country income level was also an excellent predictor (Figure 3, $\mathrm{N}=5986, \mathrm{p}<0.001$ );

157 respondents from low income countries were less likely to complete the survey than those from

158 high income countries. 
160 respondent fatigue (Figure $4, \mathrm{~N}=3642, \mathrm{p}=0.009$ ), although the relationship between app

161 importance and respondent fatigue was unusual (see Discussion). Although length of time in

162 practice had a statistically significant association with respondent fatigue (Figure $5, \mathrm{~N}=2518, \mathrm{p}$

$163=0.02$ ), the length of time in practice did not have monotonic directionality with regards to

164 respondent fatigue. There was no association between the frequency of app use and respondent

165 fatigue (Table $1, \mathrm{~N}=4659, \mathrm{p}=\mathrm{NS}$ ).

\section{Discussion}

Overall, several provider characteristics, primarily provider role and World Bank country income level, were associated with the rate of respondent fatigue for an in-app survey. Other factors that would have been assumed to be associated with less respondent fatigue, such as higher frequency of app use, turned out not to be associated. Length of time in practice and and rating of importance of the app were associated with respondent fatigue, but the relationship was not linear with respect to the ordinal categorical responses. The initial part of the following discussion will focus on addressing the details of the findings, and the implications of each of these associations, in turn. demonstrates that researchers are likely to get a higher rate of complete response from users for

177 whom the app and survey are well aligned. Physicians and anesthetists had the lowest rate of

178 fatigue, and were users most likely to interact with the subject of the survey (sugammadex) on a

179 frequent basis. Anesthesia techs and respiratory therapists are far less likely to use this drug or

180 have knowledge of it, and so the high rate of observed respondent fatigue in these user groups is 181 logical. 
183 there do not appear to be any prior studies examining respondent fatigue for in-app mHealth

184 surveys. Prior work examining respondent fatigue on the basis of unreturned surveys have found

185 a highly variable rate of responsiveness, with response rates from physicians via Web-based

186 methods as low as 45\% (Leece et al., 2004) and as high as 78\%. (Tran \& Dilley, 2010)

187 Interestingly, there have been mixed findings as to the utility of Web-based methods over paper188 and-pencil methods (Leece et al., 2004; Nicholls et al., 2011) although it is likely that the much 189 older 2004 study from Leece et al reflects a different era of connectedness. Given how much

190 easier it is to quickly click through a survey on a mobile device as compared to filling out a pen-

191 and-paper survey, or even sit down to a web survey provided via weblink, there would be no $a$

192 priori reason to assume that respondent fatigue rates would be comparable. It is now estimated

193 that two-thirds of time spent in the digital realm is time spent in mobile apps. (Bolton, 2016) On

194 the other hand, mobile apps are typically used in very short bursts, 2-8 minutes per session.

195 (“Average mobile app category session length 2015 | Statistic”) Apps, small programs with very

196 specialized functions, are likely to be launched only when practically needed, potentially limiting

197 the likelihood of participation in extraneous tasks such as in-app surveys.

198 Second, this study examines the rates of respondent fatigue during the course of a single

199 survey, administered one question at a time, with full participant control over when to cease

200 answering questions. Existing studies have primarily looked at global respondent fatigue in terms

201 of e.g. rates of survey return. By allowing participants full control, the metadata revealed a more

202 complete picture of the associations with respondent fatigue during the course of a single survey,

203 without needing to isolate phenomena such as straight-line answering. 
205 ability to reach and obtain feedback from users in resource-limited settings is a powerful promise 206 of the global adoption of smartphones and mobile apps. Perhaps resource-limitations contributed

207 the relatively high rate of respondent fatigue in users from lower-income countries: lack of access to reliable Internet connectivity (i.e. responses were recorded on the local device but not uploaded to the cloud), more expensive mobile data, and perhaps more time spent on patient care rather than in-app surveys. Another factor may be related to the expense of sugammadex itself; users from low-income countries were less likely to indicate access to sugammadex (Table 2,

212 low $=46 \%$, lower middle $=38 \%$, upper middle $=56 \%$, high income $=57 \%$, and perhaps even

213 users with access to it in lower-income countries did not feel they had enough experience with

214 the drug to complete the survey.

216 a monotonic trend, nor does it follow a pattern that would fit standard assumptions (Figure 4). It

217 is predictable that those viewing the app as "Not important at all" would have the highest rate of

218 respondent fatigue, consistent with the present findings. Not intuitive, however, is the finding

219 that users who rate the app as having average/little importance have the lowest rates of fatigue

220 (meaning there was the highest rate of survey completion by those who rated the app as of

221 middling importance to their practice). Perhaps those users who rate the app as more important to

222 their practice take less time to complete the in-app survey because when they are using the app,

223 they generally launch it for practical purposes. Likewise the association between length of

224 practice and respondent fatigue does not follow a monotonic trend, which perhaps limits the

225 usefulness of this finding in practice. It does suggest that the rate of responsiveness from

226 providers early in their practice or with many years in practice may be reduced. 
Premature termination of the survey was the approach used to measure survey fatigue for

this study. This approach was chosen because this was an objective binary outcome that was

straightforward to measure. For future studies, it may be possible to develop alternative metrics

230 of fatigue including a measurement of the rate of attrition at each question (were there step-offs?)

231 or assessment of reduced thoughtfulness (was there straight-line answering?). This may lead to a

232 conceptualization of survey fatigue on a spectrum rather than as a binary outcome. The attrition

233 approach has been previously described for Web-based surveys. (Hochheimer et al., 2016) More

234 detailed analysis of step offs and straight-line answering may provide feedback for how to

235 modify a survey to reduce the rate of fatigue. It isn't immediately clear how to objectively assess

236 straight-line responses and more work will need to be performed to analyze and characterize

237 these phenomena.

238 One limitation of these results is the lack of information about those respondents who

239 chose to opt out of the study. Ethically, no demographic information about this population was

240 possible. Those opting out of the study could be systematically biased in some way. Another

241 limitation is that the survey topic was closely aligned with the subject area of the app.

242 Respondent fatigue rates are likely to change dramatically if the topic does not align closely.

243 This is supported in some ways by this data; as noted above, users who may have had less cause

244 to use or interact with the drug were observed to have a much higher rate of respondent fatigue.

245 The effect on response rates was dramatic; fatigue rates climbed to $60 \%$ for respiratory therapists

246 who, one could speculate, would have less cause to interact with or administer sugammadex.

247 Overall, however, the population of users of this app are a self-selected group of

248 providers with enough interest in anesthesiology management to download and use an app called

249 "Anesthesiologist." Survey fatigue was measured only for those users who indicated that they 
250 had access to the drug and that it was relevant to their clinical practice (see Table S2; only users

251 who answered "Yes" to Q-02 were presented the remainder of the survey, and fatigue was

252 measured on the basis of answering Q-03 but not Q-10). Users who indicated it was not relevant

253 to their practice ("Yes, but not relevant to my practice") were not presented Q-03 through Q-10

254 and were therefore de facto excluded from the present study group.

255 The approach to the analysis was to perform univariable regression on each factor rather

256 than multiple regression. Multiple regression was avoided due to missingness in the data at a rate

257 high enough that complete case regression of the dataset may yield biased responses, and also the

258 rate of missingness may have yielded biased results following multiple imputation. Our concern

259 is that if the missingness is not at random, then imputation would be an inappropriate approach

260 as it would bias the sample in a similar manner to a complete case analysis. This missingness

261 resulted from the approach to the demographic survey deployment, in which some questions

262 were delayed in presentation to reduce the burden of the total survey load when initially opting

263 into the study. This approach carried the benefit of reducing this burden but also introduced

264 fatigue into the demographic survey itself. The presence of respondent fatigue in the

265 demographic survey itself could result in non-random differences in populations exhibiting

266 respondent fatigue at the sugammadex survey level. There is a hint that the missingness is

267 nonrandom in that the raw respondent fatigue rates per category in the univariable analysis are

268 different from one another -- as high as $34 \%$ overall for country income level and as low as $24 \%$

269 for community served. If there is non-random missingness, then it becomes much more fraught

270 to perform multiple variable regression due to an inability to account for this confounder. This is

271 a limitation of this data set that potentially also limits the generalizability of the findings.

\section{Conclusions}


274 mobile apps, which can serve as powerful platforms for reaching a global set of users, studying

275 practice patterns and usage habits. Studies should be carefully designed to mitigate fatigue as

276 well as powered with the understanding of the respondent characteristics that may have higher

277 rates of respondent fatigue. Variable rates of respondent fatigue across different categories of

278 providers should be expected. The use of large-scale analytics will likely continue to grow,

279 leading to crowdsourced sources of information. For example, researchers may use trend data

280 from the from Google searches or from in-app clicks and surveys to detect outbreaks of disease.

281 Other future studies may also survey providers about post-marketing drug-related adverse events.

282 The ability to predict response rates, and therefore power these studies, will rely on an

283 understanding of what factors may influence survey fatigue. The work presented here should

284 help to elucidate some of that factors that influence respondent fatigue, as well as demonstrate

285 the applicability of this methodology to measure these fatigue rates for in-app surveys for 286 providers using mHealth apps.

\section{Acknowledgements}

288 I'd like to thank George Easton, who developed the methodology for calculating frequency of

289 app use but far more importantly has provided excellent conversation and discussion that has

290 influenced my thinking leading to this work. I'd also like to thank Scott Gillespie, who provided

291 the R code for mapping provider country of origin income level and helped me with my

292 statistical thinking.

293

294 References

2952015 Joint Statistical Meetings - Statistics: Making Better Decisions. - Seattle, Washington. 
296

297

298

299

300

301

302

303

304

305

306

307

308

309

310

311

312

313

314

315

316

317

318

Available at

https://ww2.amstat.org/meetings/jsm/2015/onlineprogram/AbstractDetails.cfm?abstractid= 316392 (accessed June 17, 2017).

Akter S., Ray P. 2010. mHealth - an Ultimate Platform to Serve the Unserved. Yearbook of medical informatics:94-100.

Analytics for Xamarin. Available at http://www.webcitation.org/6im0la9R0 (accessed July 5, 2016).

Arel-Bundock V. 2014. countrycode: Convert Country Names and Country Codes.

Average mobile app category session length 2015 | Statistic. Available at http://www.webcitation.org/6oocYFt39 (accessed March 8, 2017).

Ben-Nun P. 2008. Respondent fatigue. Encyclopedia of survey research methods 2:742-743.

Bolton D. 2016.Two-Thirds Of All Digital Time Is Now Spent On A Mobile Device - ARC. Available at http://www.webcitation.org/6pxulGKhY (accessed March 8, 2017).

Cook DA., Wittich CM., Daniels WL., West CP., Harris AM., Beebe TJ. 2016. Incentive and Reminder Strategies to Improve Response Rate for Internet-Based Physician Surveys: A Randomized Experiment. Journal of medical Internet research 18:e244. DOI: 10.2196/jmir.6318.

Cull WL., O’Connor KG., Sharp S., Tang S-FS. 2005. Response rates and response bias for 50 surveys of pediatricians. Health services research 40:213-226. DOI: 10.1111/j.14756773.2005.00350.x.

Definitions of mHealth 2012. Available at http://www.himss.org/definitions-mhealth (accessed August 4, 2017).

FDA., U.S. Department of Health and Human Services, Food and Drug Administration, Center 
319 for Devices and Radiological Health, Center for Biologics Evaluation and Research.

320 2015.Mobile Medical Applications: Guidance for Industry and Food and Drug

321 Administration Staff. Available at http://www.webcitation.org/6lZhlyUag (accessed

322 October 27, 2016).

323 Glidewell L., Thomas R., MacLennan G., Bonetti D., Johnston M., Eccles MP., Edlin R., Pitts

324 NB., Clarkson J., Steen N., Grimshaw JM. 2012. Do incentives, reminders or reduced

325 burden improve healthcare professional response rates in postal questionnaires? two

326 randomised controlled trials. BMC health services research 12:250. DOI: 10.1186/1472-

$327 \quad 6963-12-250$.

328 Google Analytics. Available at http://www.webcitation.org/6im0miwiZ (accessed July 5, 2016).

329 Harris PA., Taylor R., Thielke R., Payne J., Gonzalez N., Conde JG., Others. 2009. A metadata-

330 driven methodology and workflow process for providing translational research informatics

331 support. Journal of biomedical informatics 42:377-381.

332 Hochheimer CJ., Sabo RT., Krist AH., Day T., Cyrus J., Woolf SH. 2016. Methods for

333 Evaluating Respondent Attrition in Web-Based Surveys. Journal of medical Internet

334 research 18:e301. DOI: 10.2196/jmir.6342.

335 Introducing Amazon Mobile Analytics. Available at http://www.webcitation.org/6im0lxPCw

336 (accessed July 5, 2016).

337 JSM 2016 Online Program. Available at

338 https://ww2.amstat.org/meetings/jsm/2016/onlineprogram/AbstractDetails.cfm?abstractid=

339319908 (accessed June 17, 2017).

340 Kellerman S. 2001. Physician response to surveys A review of the literature. American journal of

341 preventive medicine 20:61-67. DOI: 10.1016/S0749-3797(00)00258-0. 
342 Leece P., Bhandari M., Sprague S., Swiontkowski MF., Schemitsch EH., Tornetta P., Devereaux

343 PJ., Guyatt GH. 2004. Internet versus mailed questionnaires: a controlled comparison (2).

344 Journal of medical Internet research 6:e39. DOI: 10.2196/jmir.6.4.e39.

345 Liu C., Chang L., Qing Z., Holroyd KA., Seng EK. 2011. Status and trends of mobile-health

346 applications for iOS devices: A developer's perspective. The Journal of systems and

347 software 84:2022-2033.

348 MeSH Browser. Available at https://meshb.nlm.nih.gov/record/ui? ui=D017216 (accessed

$349 \quad$ August 4, 2017).

350 Microsoft Azure. Available at http://www.webcitation.org/6im0mCbew (accessed July 5, 2016).

351 Nicholls K., Chapman K., Shaw T., Perkins A., Sullivan MM., Crutchfield S., Reed E. 2011.

352 Enhancing response rates in physician surveys: the limited utility of electronic options.

353 Health services research 46:1675-1682. DOI: 10.1111/j.1475-6773.2011.01261.x.

354 Number of apps available in leading app stores 2015 | Statistic. Available at

355 http://www.webcitation.org/6dPd06FCB (accessed August 19, 2015).

356 Ooms J. 2014. The jsonlite Package: A Practical and Consistent Mapping Between JSON Data

357 and R Objects. arXiv:1403. 2805 [stat. CO].

358 O'Reilly-Shah VN. 2016. A Global Survey of Sugammadex Practice Patterns Indicates High

359 Rates of Selective Administration and Lower than Expected Adverse Event Rates.

360 O’Reilly-Shah V., Easton G., Gillespie S. 2017. Assessing the global usage and value of a

361 mobile healthcare app using large-scale analytics. BMJ Global Health (in press). DOI:

$362 \quad$ 10.1136/bmjgh-2017-000299.

363 O’Reilly-Shah V., Mackey S. 2016. Survalytics: An Open-Source Cloud-Integrated Experience

364 Sampling, Survey, and Analytics and Metadata Collection Module for Android Operating 
365 System Apps. JMIR mHealth and uHealth 4:e46. DOI: 10.2196/mhealth.5397.

366 O’Reilly-Shah VN., Wolf FA., Jabaley CS., Lynde GC. 2017. Using a worldwide in-app survey

367 to explore sugammadex usage patterns: a prospective observational study. British Journal of

368 Anaesthesia (in press). DOI: 10.1093/bja/aex171.

369 Ozdalga E., Ozdalga A., Ahuja N. 2012. The smartphone in medicine: a review of current and

370 potential use among physicians and students. Journal of medical Internet research 14:e128.

$371 \quad$ DOI: 10.2196/jmir.1994.

372 R Core Team. 2015.R: A language and environment for statistical computing [Internet]. Vienna,

373 Austria: R Foundation for Statistical Computing; 2015 (accessed 2015).

374 Rivera J., van der Meulen R.Gartner says annual smartphone sales surpassed sales of feature

375 phones for the first time in 2013 (feb. 2014). Available at

376 http://www.webcitation.org/6dPWAR51k (accessed November 29, 2015).

377 RStudio-Team. 2015.RStudio Team. Available at https://www.rstudio.com/ (accessed August 4, $378 \quad 2017)$.

379 South A. 2011. rworldmap: A New R package for Mapping Global Data. The R Journal 3:35-43.

380 Stoyanov SR., Hides L., Kavanagh DJ., Zelenko O., Tjondronegoro D., Mani M. 2015. Mobile

381 app rating scale: a new tool for assessing the quality of health mobile apps. JMIR mHealth 382 and uHealth 3:e27. DOI: 10.2196/mhealth.3422.

383 Tran N., Dilley JA. 2010. Achieving a high response rate with a health care provider survey, 384 Washington State, 2006. Preventing chronic disease 7:A111.

385 Whelan T. Antecedents of anonymity perceptions in web-based surveys. PsycEXTRA Dataset.

386 DOI: $10.1037 / \mathrm{e} 518442013-442$.

387 World Bank Country Incomes. Available at http://www.webcitation.org/6lYLKtdpy (accessed 
$388 \quad$ October 26, 2016).

389 Xiong H., Huang Y., Barnes LE., Gerber MS. Sensus: A Cross-Platform, General-Purpose

390 System for Mobile Crowdsensing in Human-Subject Studies. ptl.sys.virginia.edu.

391

392 
393 Table 1: Univariable logisticregression results examining the association between various

394 independent variables and the presence of respondent fatigue. The association of respondent

395 fatigue with provider role, country income level, rating of app importance, length of time in

396 practice, and frequency of app use are discussed in greater detail in the text.

\begin{tabular}{|c|c|c|c|c|c|c|c|c|c|c|}
\hline \multirow[b]{2}{*}{ Provider Role } & \multirow{2}{*}{$\begin{array}{c}\text { N (Users) } \\
5333\end{array}$} & \multirow[t]{2}{*}{\begin{tabular}{c|}
$\mathrm{N}$ (Fatigued) \\
1708 \\
\end{tabular}} & \multirow{2}{*}{$\begin{array}{c}\text { Raw Proportion of } \\
\text { Respondents With } \\
\text { Survey Fatigue } \\
\text { (\%) } \\
32 \% \\
\end{array}$} & \multicolumn{3}{|c|}{$\begin{array}{l}\text { Odds Ratio of Being Fatigued } \\
\text { Compared to Referent Category and } \\
95 \% \text { Confidence Interval }\end{array}$} & \multicolumn{3}{|c|}{$\begin{array}{c}\text { Proportion of Respondents With Survey } \\
\text { Fatigue } \\
\text { (Estimated Percentage and } 95 \% \\
\text { Confidence Interval) }\end{array}$} & \multirow{3}{*}{$\begin{array}{c}\text { Univariable } \\
\text { p-value } \\
\text { (Overall Wald Per Ind. Var } \\
\text { / } \\
\text { vs Reference Category) } \\
<0.001\end{array}$} \\
\hline & & & & & & & & Overall & -value $=$ & \\
\hline Physician & 1832 & 467 & $25 \%$ & Referent & Referent & Referent & $25 \%$ & $24 \%$ & $28 \%$ & \\
\hline PhysicianTrainee & 1331 & 343 & $26 \%$ & 1.0 & 0.9 & 1.2 & $26 \%$ & $23 \%$ & $28 \%$ & 0.85 \\
\hline AA or CRNA & 1488 & 553 & $37 \%$ & 1.7 & 1.5 & 2.0 & $37 \%$ & $35 \%$ & $40 \%$ & $<0.001$ \\
\hline AnesthesiaTechnician & 324 & 171 & $53 \%$ & 3.3 & 2.6 & 4.2 & $53 \%$ & $47 \%$ & $58 \%$ & $<0.001$ \\
\hline AA or CRNA Trainee & 210 & 88 & $42 \%$ & 2.1 & 1.6 & 2.8 & $42 \%$ & $35 \%$ & $49 \%$ & $<0.001$ \\
\hline Technically TrainedinAnesthesia & 75 & 40 & $53 \%$ & 3.3 & 2.1 & 5.3 & $53 \%$ & $42 \%$ & $64 \%$ & $<0.001$ \\
\hline Respiratory Therapist & 73 & 46 & $63 \%$ & 5.0 & 3.1 & 8.2 & $63 \%$ & $52 \%$ & $73 \%$ & $<0.001$ \\
\hline Country Income & 5988 & 2057 & $34 \%$ & & & & & \multicolumn{2}{|c|}{ Overall Wald $p$-value $=$} & $<0.001$ \\
\hline Low income & 160 & 85 & $53 \%$ & Referent & Referent & Referent & $53 \%$ & $45 \%$ & $61 \%$ & Referent \\
\hline Lower middle income & 1172 & 548 & $47 \%$ & 0.8 & 0.6 & 1.1 & $47 \%$ & $44 \%$ & $50 \%$ & 0.13 \\
\hline Upper middle income & 1981 & 779 & $39 \%$ & 0.6 & 0.4 & 0.8 & $39 \%$ & $37 \%$ & $41 \%$ & $<0.001$ \\
\hline High income & 2675 & 645 & $24 \%$ & 0.3 & 0.2 & 0.4 & $24 \%$ & $23 \%$ & $26 \%$ & $<0.001$ \\
\hline Rating of App Importance & 3642 & 956 & $26 \%$ & & & & & \multicolumn{2}{|c|}{ Overall Wald $p$-value $=$} & $<0.001$ \\
\hline Absolutely Essential & 422 & 142 & $34 \%$ & Referent & Referent & Referent & $34 \%$ & $29 \%$ & $38 \%$ & Referent \\
\hline Very Important & 1174 & 306 & $26 \%$ & 0.7 & 0.5 & 0.9 & $26 \%$ & $24 \%$ & $29 \%$ & 0.003 \\
\hline Of Average Importance & 1123 & 230 & $20 \%$ & 0.5 & 0.4 & 0.7 & $20 \%$ & $18 \%$ & $23 \%$ & $<0.001$ \\
\hline Of Little Importance & 473 & 101 & $21 \%$ & 0.5 & 0.4 & 0.7 & $21 \%$ & $18 \%$ & $25 \%$ & $<0.001$ \\
\hline Not Important At All & 450 & 177 & $39 \%$ & 1.3 & 1.0 & 1.7 & $39 \%$ & $35 \%$ & $44 \%$ & 0.082 \\
\hline Length of Time in Practice & 2518 & 685 & $27 \%$ & & & & & \multicolumn{2}{|c|}{ Overall Wald p-value $=$} & $<0.001$ \\
\hline $0-5$ Years & 1030 & 285 & $28 \%$ & Referent & Referent & Referent & $28 \%$ & $25 \%$ & $30 \%$ & Referent \\
\hline $6-10$ Years & 489 & 99 & $20 \%$ & 0.7 & 0.5 & 0.9 & $20 \%$ & $17 \%$ & $24 \%$ & 0.002 \\
\hline $11-20$ Years & 448 & 109 & $24 \%$ & 0.8 & 0.6 & 1.1 & $24 \%$ & $21 \%$ & $28 \%$ & 0.18 \\
\hline $21-30$ Years & 551 & 192 & $35 \%$ & 1.4 & 1.1 & 1.7 & $35 \%$ & $31 \%$ & $39 \%$ & 0.003 \\
\hline Anesthesia Practice Model & 2951 & 709 & $24 \%$ & & & & & \multicolumn{2}{|c|}{ Overall Wald p-value $=$} & 0.003 \\
\hline Physician only & 1040 & 249 & $24 \%$ & Referent & Referent & Referent & $24 \%$ & $21 \%$ & $27 \%$ & Referent \\
\hline $\begin{array}{l}\text { Physician supervised, anesthesiologist } \\
\text { on site }\end{array}$ & 1292 & 276 & $21 \%$ & 0.9 & 0.7 & 1.0 & $21 \%$ & $19 \%$ & $24 \%$ & 0.14 \\
\hline $\begin{array}{c}\text { Physician supervised, } \\
\text { non-anesthesiologist physician on site }\end{array}$ & 189 & 55 & $29 \%$ & 1.3 & 0.9 & 1.8 & $29 \%$ & $23 \%$ & $36 \%$ & 0.13 \\
\hline $\begin{array}{l}\text { Physician supervised, no physician on } \\
\text { site }\end{array}$ & 117 & 34 & $29 \%$ & 1.3 & 0.8 & 2.0 & $29 \%$ & $21 \%$ & $38 \%$ & 0.22 \\
\hline No physician supervision & 170 & 46 & $27 \%$ & 1.2 & 0.8 & 1.7 & $27 \%$ & $21 \%$ & $34 \%$ & 0.38 \\
\hline Not an anesthesia provider & 143 & 49 & $34 \%$ & 1.7 & 1.1 & 2.4 & $34 \%$ & $27 \%$ & $42 \%$ & 0.008 \\
\hline Practice Type & 3113 & 770 & $25 \%$ & & & & & \multicolumn{2}{|c|}{ Overall Wald $p$-value $=$} & $<0.001$ \\
\hline Private clinic or office & 567 & 211 & $37 \%$ & Referent & Referent & Referent & $37 \%$ & $33 \%$ & $41 \%$ & Referent \\
\hline Local health clinic & 277 & 88 & $32 \%$ & 0.8 & 0.6 & 1.1 & $32 \%$ & $26 \%$ & $37 \%$ & 0.12 \\
\hline Ambulatory surgery center & 133 & 49 & $37 \%$ & 1.0 & 0.7 & 1.5 & $37 \%$ & $29 \%$ & $45 \%$ & 0.94 \\
\hline Small community hospital & 330 & 65 & $20 \%$ & 0.4 & 0.3 & 0.6 & $20 \%$ & $16 \%$ & $24 \%$ & $<0.001$ \\
\hline Large community hospital & 932 & 183 & $20 \%$ & 0.4 & 0.3 & 0.5 & $20 \%$ & $17 \%$ & $22 \%$ & $<0.001$ \\
\hline Academic department/University hospital & 874 & 174 & $20 \%$ & 0.4 & 0.3 & 0.5 & $20 \%$ & $17 \%$ & $23 \%$ & $<0.001$ \\
\hline Practice Size & 3342 & 880 & $26 \%$ & & & & & \multicolumn{2}{|c|}{ Overall Wald $p$-value $=$} & $<0.001$ \\
\hline Solo & 1364 & 426 & $31 \%$ & Referent & Referent & Referent & $31 \%$ & $29 \%$ & $34 \%$ & Referent \\
\hline Small Group Less Than 10 & 695 & 223 & $32 \%$ & 1.0 & 0.9 & 1.3 & $32 \%$ & $29 \%$ & $36 \%$ & 0.69 \\
\hline Medium Group 10-25 & 396 & 63 & $16 \%$ & 0.4 & 0.3 & 0.6 & $16 \%$ & $13 \%$ & $20 \%$ & $<0.001$ \\
\hline Large Group Greater Than 25 & 887 & 168 & $19 \%$ & 0.5 & 0.4 & 0.6 & $19 \%$ & $16 \%$ & $22 \%$ & $<0.001$ \\
\hline Community Served & 2256 & 552 & $24 \%$ & & & & & \multicolumn{2}{|c|}{ Overall Wald p-value $=$} & $<0.001$ \\
\hline Rural & 538 & 177 & $33 \%$ & Referent & Referent & Referent & $33 \%$ & $29 \%$ & $37 \%$ & Referent \\
\hline Suburban & 374 & 91 & $24 \%$ & 0.7 & 0.5 & 0.9 & $24 \%$ & $20 \%$ & $29 \%$ & 0.01 \\
\hline Urban & 1344 & 284 & $21 \%$ & 0.5 & 0.4 & 0.7 & $21 \%$ & $19 \%$ & $23 \%$ & $<0.001$ \\
\hline Frequency of App Use & & & & & & & & \multicolumn{2}{|c|}{ Overall Wald p-value $=$} & 0.44 \\
\hline
\end{tabular}


401 Table 2: Number and percentage of responses from users of each country income category within

402 each category of responses regarding access to sugammadex.

403

\begin{tabular}{|c|c|c|c|c|c|c|c|c|}
\hline & \multicolumn{2}{|c|}{ Low income } & \multicolumn{2}{|c|}{ Lower middle income } & \multicolumn{2}{|c|}{ Upper middle income } & \multicolumn{2}{|c|}{ High income } \\
\hline & $\mathrm{N}$ & $\%$ & $\mathrm{~N}$ & $\%$ & $\mathrm{~N}$ & $\%$ & $\mathrm{~N}$ & $\%$ \\
\hline Yes & 176 & $46 \%$ & 1294 & $38 \%$ & 2107 & $56 \%$ & 2745 & $57 \%$ \\
\hline No, not approved in my country & 84 & $22 \%$ & 760 & $22 \%$ & 544 & $14 \%$ & 436 & $9 \%$ \\
\hline No, not on formulary & 57 & $15 \%$ & 775 & $23 \%$ & 613 & $16 \%$ & 956 & $20 \%$ \\
\hline Yes, but not relevant to my practice & 23 & $6 \%$ & 222 & $6 \%$ & 250 & $7 \%$ & 233 & $5 \%$ \\
\hline No or unsure, but not relevant to my practice & 40 & $11 \%$ & 366 & $11 \%$ & 257 & $7 \%$ & 406 & $9 \%$ \\
\hline
\end{tabular}

404

405

406

407

408 
409 Figure 1: Data collected over the study period, including visualization of the difference between

410 rates of response to the first unbranched sugammadex survey question (Q-03) and the final

411 sugammadex survey question (Q-10).

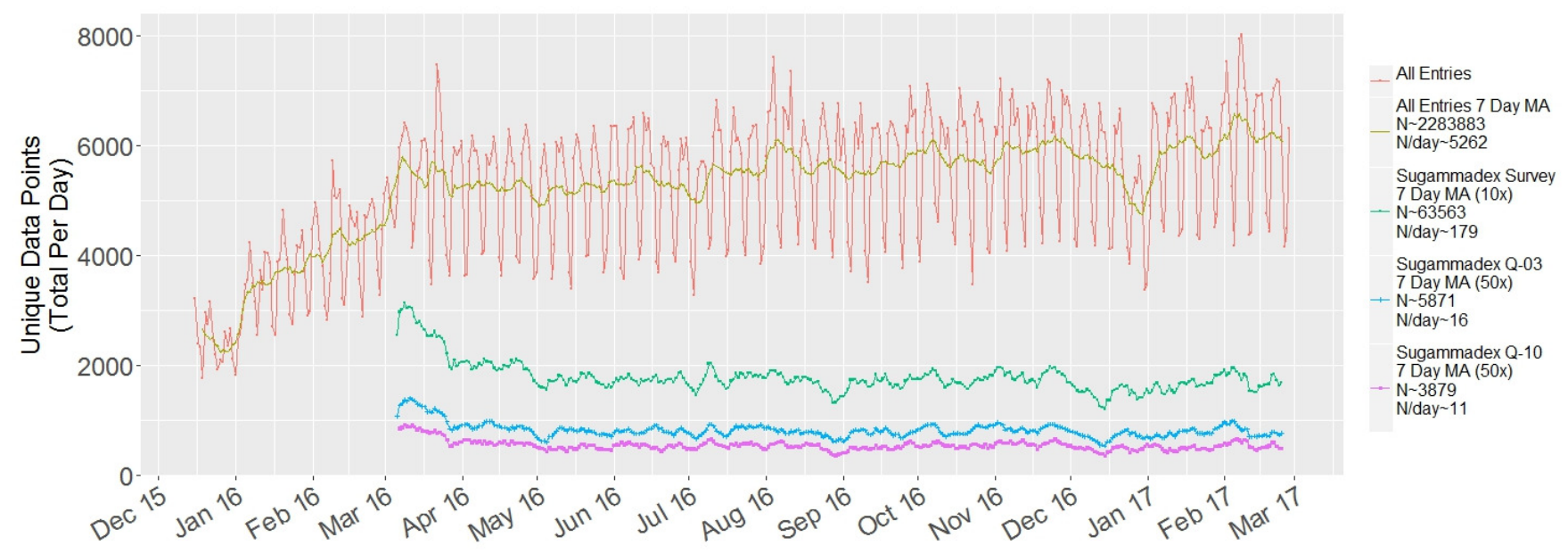

412

413

414 
415 Figure 2: Observed fatigue rate versus provider role. Top number is the number of participants

416 with respondent fatigue (see Methods). Bottom number is the total number of participants in the

417 category.

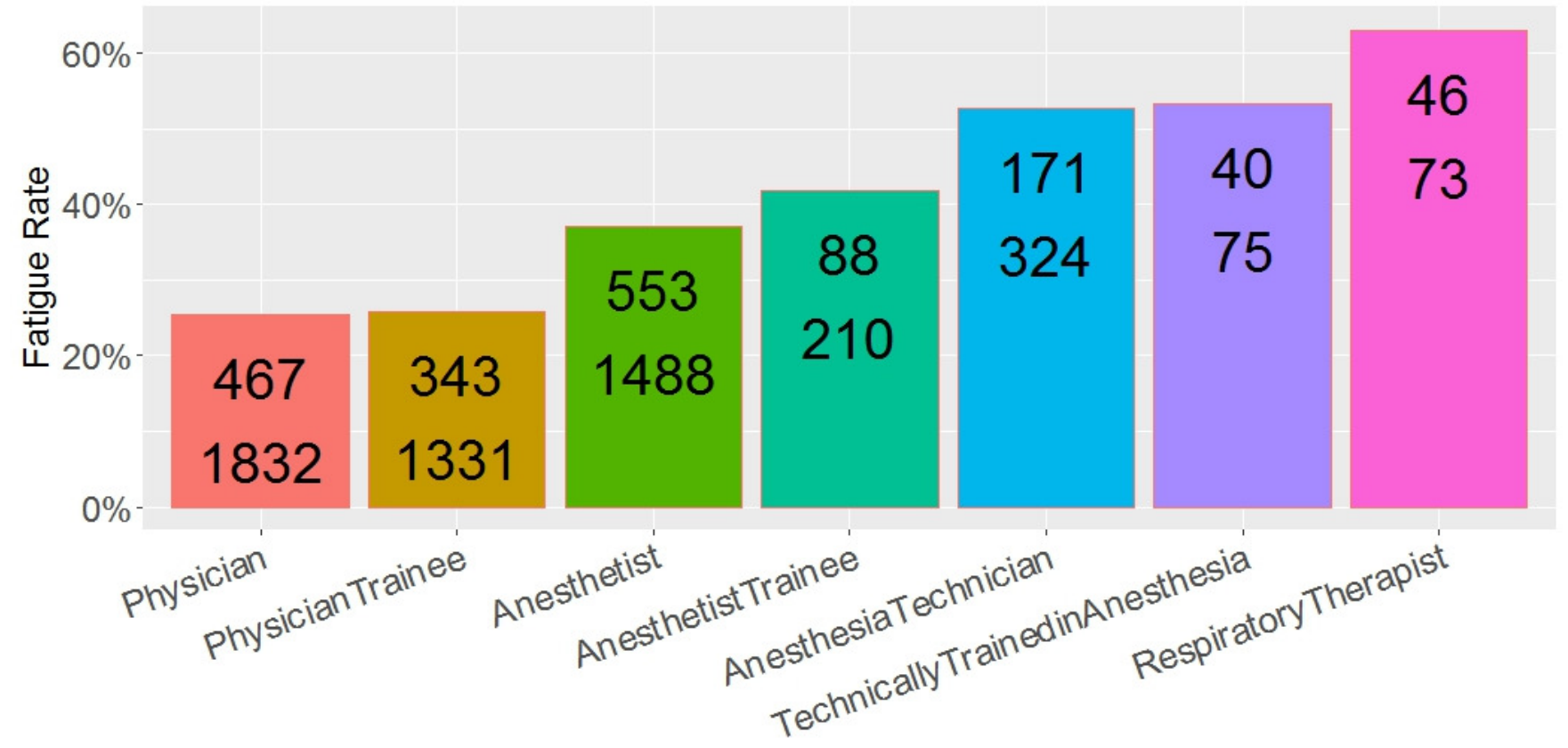

418

419

420

421

422 
423 Figure 3: Observed fatigue rate versus primary country World Bank income level. Top number is

424 the number of participants with respondent fatigue (see Methods). Bottom number is the total 425 number of participants in the category.

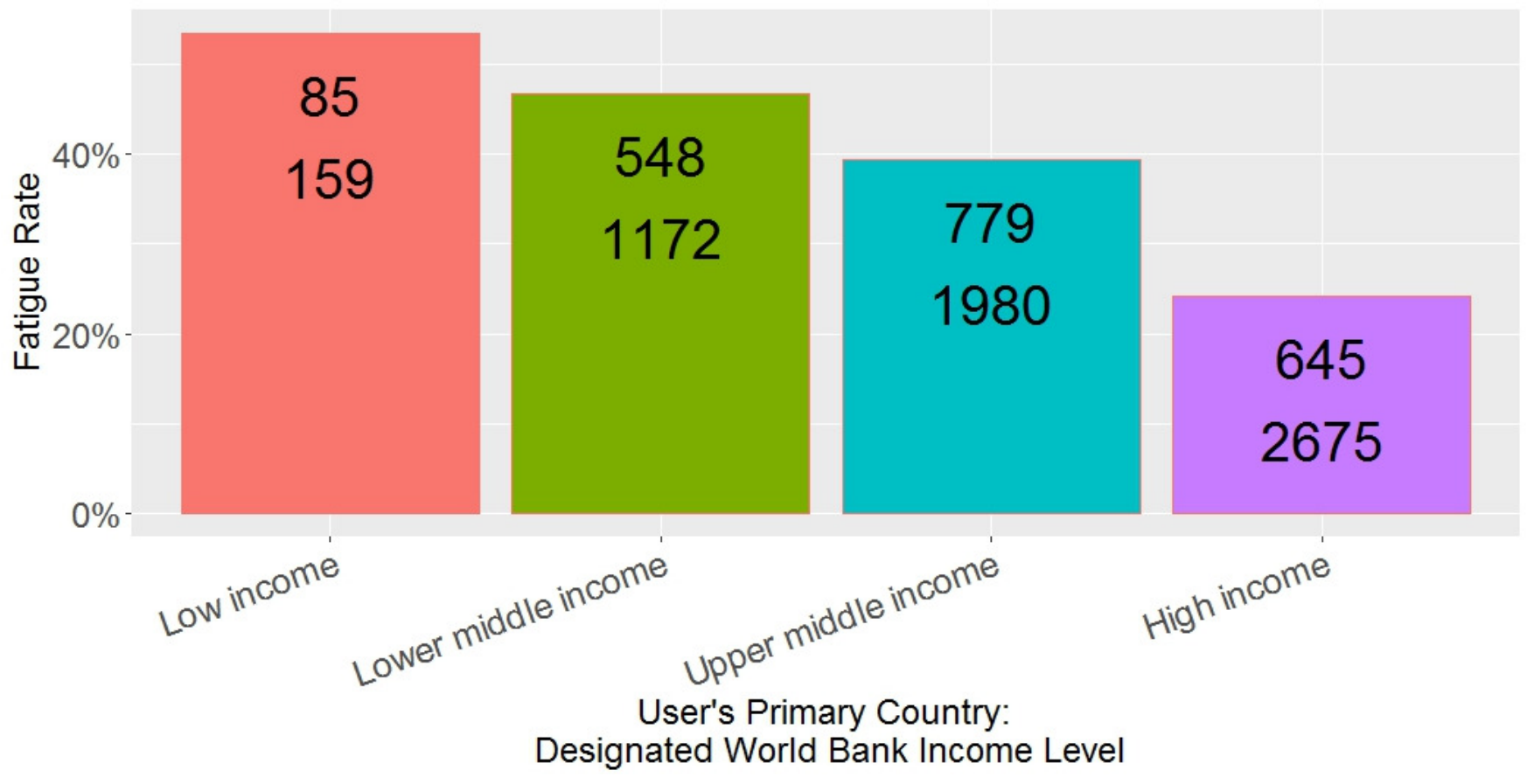

427

428

429 
430 Figure 4: Observed fatigue rate versus provider rating of app importance. Top number is the

431 number of participants with respondent fatigue (see Methods). Bottom number is the total

432 number of participants in the category.

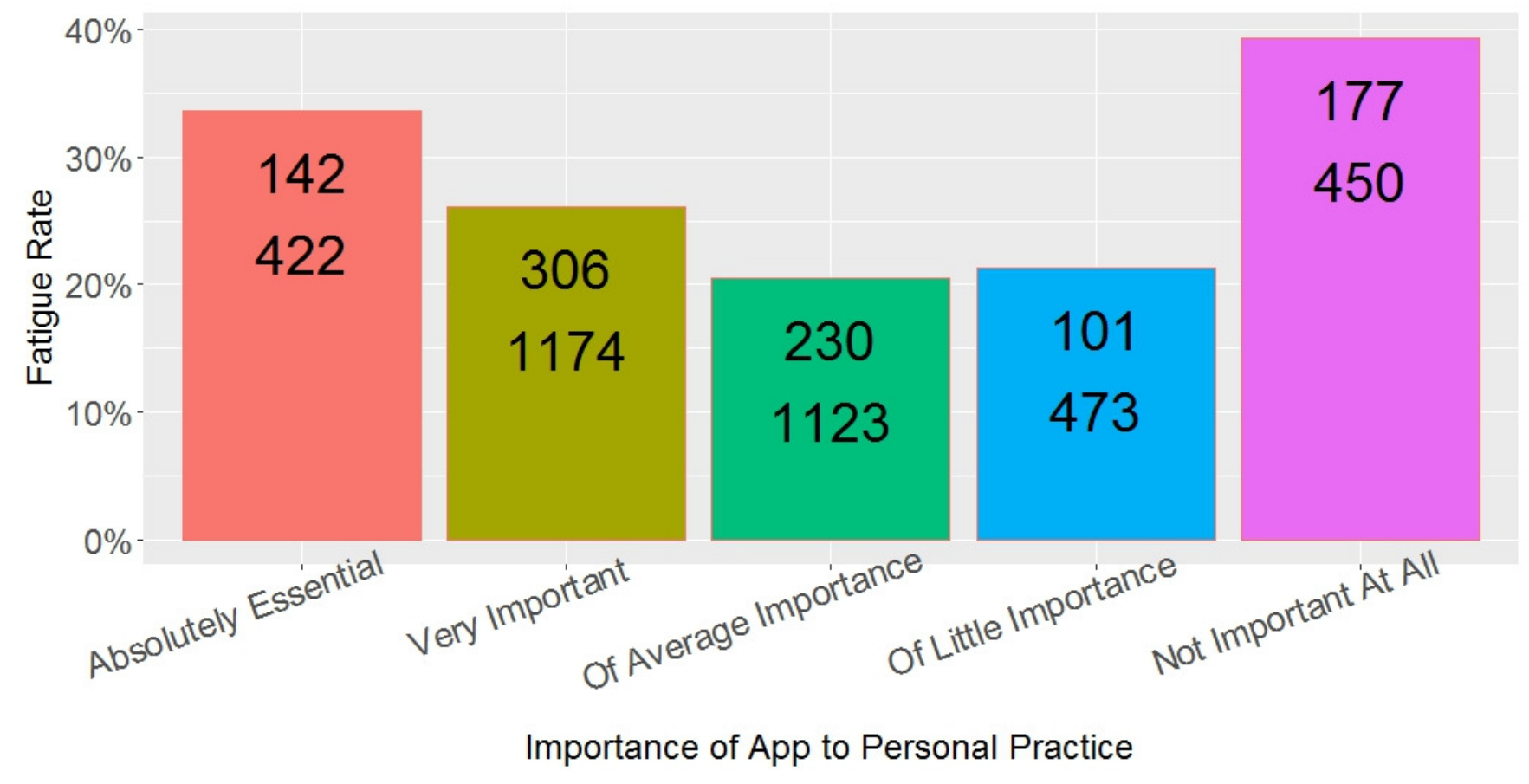

434

435

436 
437 Figure 5: Observed fatigue rate versus provider length of time in practice. Top number is the

438 number of participants with respondent fatigue (see Methods). Bottom number is the total

439 number of participants in the category.

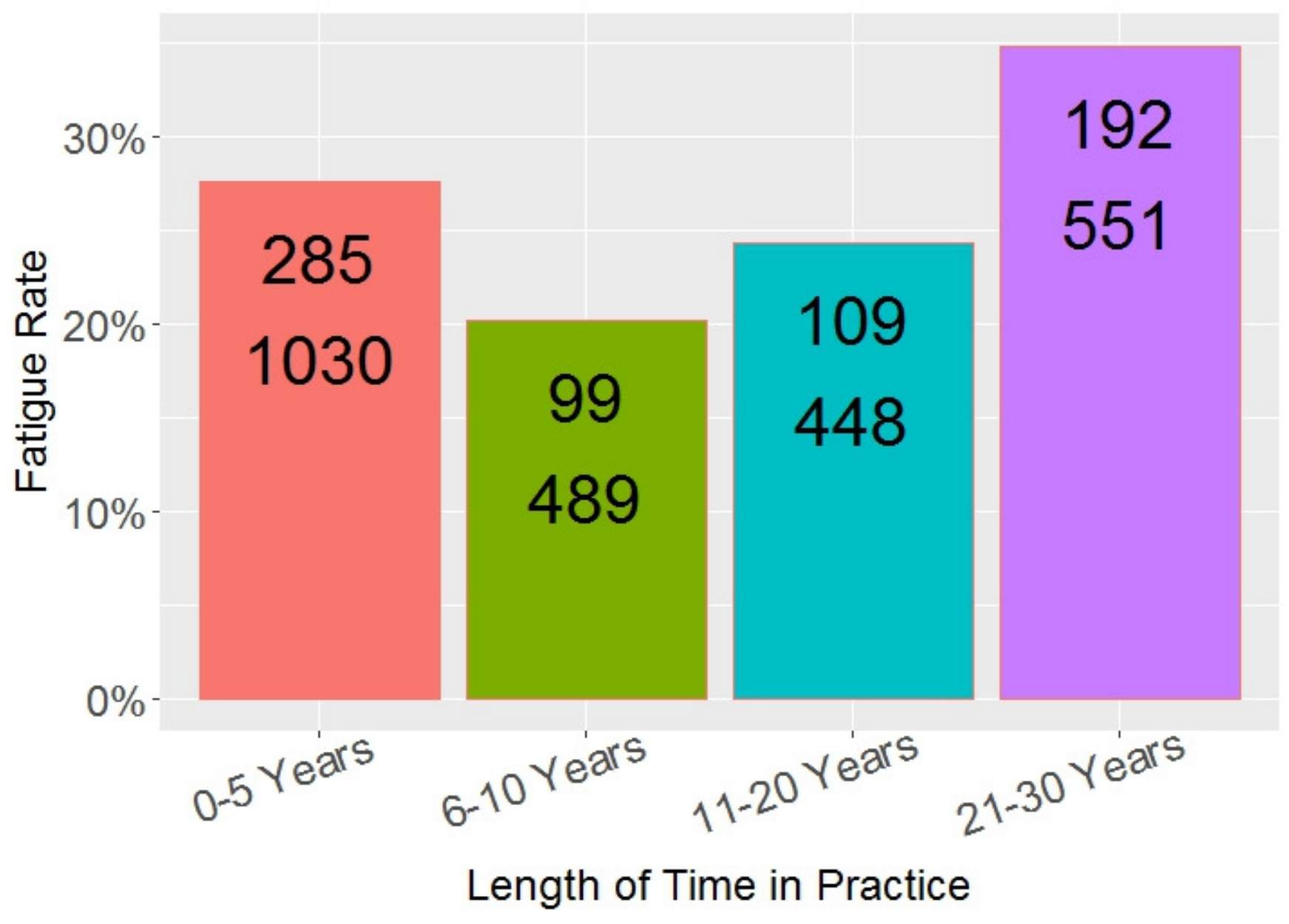

441

442

443

444 